\title{
Cross-Continental Dispersal of Major HIV-1 CRF01_AE Clusters in China
}

\author{
Minghui An ${ }^{1,2,3,4}$, Xiaoxu Han 1,2,3,4, Bin Zhao ${ }^{1,2,3,4}$, Suzanne English ${ }^{5}$, Simon D. W. Frost ${ }^{6}$ \\ Hongyi Zhang ${ }^{5 *}$ and Hong Shang ${ }^{1,2,3,4 *}$ on behalf of the UK HIV Drug Resistance Database \\ ${ }^{1}$ NHC Key Laboratory of AIDS Immunology (China Medical University), Department of Laboratory Medicine, The First \\ Affiliated Hospital of China Medical University, Shenyang, China, ${ }^{2}$ National Clinical Research Center for Laboratory Medicine, \\ The First Affiliated Hospital of China Medical University, Shenyang, China, ${ }^{3}$ Key Laboratory of AIDS Immunology of Liaoning \\ Province, The First Affiliated Hospital of China Medical University, Shenyang, China, ${ }^{4}$ Key Laboratory of AIDS Immunology, \\ Chinese Academy of Medical Sciences, Shenyang, China, ${ }^{5}$ PHE Clinical Microbiology and Public Health Laboratory, \\ Addenbrooke's Hospital, Cambridge, United Kingdom, ${ }^{6}$ Department of Veterinary Medicine, University of Cambridge, \\ Cambridge, United Kingdom
}

\section{OPEN ACCESS}

Edited by:

Gkikas Magiorkinis,

National and Kapodistrian University

of Athens, Greece

Reviewed by: $\mathrm{Bin} \mathrm{Su}$

Capital Medical University, China Evangelia Georgia Kostaki, National and Kapodistrian University of Athens, Greece

*Correspondence: Hong Shang hongshang100@hotmail.com Hongyi Zhang

hongyi.zhang@addenbrookes.nhs.uk

Specialty section: This article was submitted to Virology,

a section of the journal Frontiers in Microbiology

Received: 03 September 2019 Accepted: 13 January 2020

Published: 31 January 2020

Citation:

An M, Han X, Zhao B, English S, Frost SDW, Zhang $H$ and Shang $H$ (2020) Cross-Continental Dispersal of Major HIV-1 CRF01_AE Clusters in China. Front. Microbiol. 11:61. doi: 10.3389/fmicb.2020.00061
Since the 1990s, several distinct clusters of human immunodeficiency virus-type 1 (HIV-1) CRF01_AE related to a large epidemic in China have been identified, but it is yet poorly understood whether its transmission has dispersed globally. We aimed to characterize and quantify the genetic relationship of HIV-1 CRF01_AEs circulating in China and other countries. Using representative sequences of Chinese clusters as queries, all relevant CRF01_AE pol sequences in two large databases (the Los Alamos HIV sequence database and the UK HIV Drug Resistance Database) were selected with the online basic local alignment search (BLAST) tool. Phylogenetic and phylogeographic analyses were then carried out to characterize possible linkage of CRF01_AE strains between China and the rest of the world. We identified that 269 strains isolated in other parts of the world were associated with five major Chinese CRF01_AE clusters. 80.7\% were located within CN.01AE.HST/IDU-2, most of which were born in Southeast Asia. 17.8\% were clustered with CN.01AE.MSM-4 and -5 . Two distinct sub-clusters associated with Chinese men who have sex with men (MSM) emerged in HK-United Kingdom and Japan after 2000. Our analysis suggests that HIV-1 CRF01_AE strains related to viral transmission in China were initially brought to the United Kingdom or other countries during the 1990s by Asian immigrants or returning international tourists from Southeast Asia, and then after having circulated among MSM in China for several years, these Chinese strains dispersed outside again, possibly through MSM network. This study provided evidence of regional and global dispersal of Chinese CRF01_AE strains. It would also help understand the global landscape of HIV epidemic associated with CRF01_AE transmission and highlight the need for further international collaborative study in this field.

Keywords: HIV-1, CRF01_AE, cross-continental, dispersal, China 


\section{INTRODUCTION}

The HIV-1 pandemic is currently dominated by Group M, which has diversified into nine subtypes, seven sub-subtypes (A1, A2, A3, A4, A6, F1, and F2), 98 circulating recombinant forms (CRF) and numerous unique recombinant forms (URF). Human migration, globalization, and different risk factors for transmission between hosts have shaped the geographical and demographic distribution of HIV. For example, strains of CRF01_AE and B co-circulate among high risk sexual population and injecting drug users (IDUs) in East and South-east Asia, in contrast to MSM population in Western Europe and North America, where circulating HIV-1 strains are dominated by subtype B (Peeters et al., 2013).

However, recent studies have reported the emergence and rapid increase of non-B subtypes in various populations globally (Cuevas et al., 2009; Liao et al., 2009; Hawke et al., 2013; Neogi et al., 2014; Beloukas et al., 2016; Nikolopoulos et al., 2016; Dennis et al., 2017), especially among men who have sex with men (MSM) in China (Zhang et al., 2015). CRF01_AE, CRF_BC and Subtype B/B' are most prevalent in Mainland China (Su et al., 2014), and CRF01_AE has recently replaced subtype $\mathrm{B}$ as the dominant circulating subtype among Chinese MSM (Zhang et al., 2015). A number of detailed phylogenetic analyses revealed that several major transmission clusters of HIV-1 CRF01_AE existed and expanded after their multiple introductions into China (Abubakar et al., 2013; Feng et al., 2013; Peng et al., 2015; Zeng et al., 2016; Wang et al., 2017). Three of them were prevalent among heterosexuals and IDUs (CN.01AE.HST/IDU-1/2/3 clusters), and two were related to MSM transmission (CN.01AE.MSM-4/5 clusters) (Feng et al., 2013). By contrast, in Western Europe and North America, subtype $B$ was the early epidemic strain and now is still dominating (Hemelaar, 2013). However, some studies also reported that the frequency of non- $B$ subtypes has been increasing in Western Europe and North America (Thomson and Najera, 2007; UK Collaborative Group on HIV Drug Resistance, 2014), especially among MSM population of eight countries in North America, Western Europe and Australia (Sullivan et al., 2009). Recent studies of European HIV populations have reported that CRF01_AE strains have emerged in European MSM population, accounting for $11.2 \%$ in the United Kingdom (Fox et al., 2010) and $11.1 \%$ in Spain (Cuevas et al., 2009), among newly detected non-B strains. However, few studies have focused on the genetic links of HIV-1 CRF01_AE strains between countries or regions.

While several distinct clusters of CRF01_AE have been identified to be related to a large epidemic in Mainland China, it is poorly understood whether it has dispersed elsewhere. Therefore, the aim of this study was to characterize and quantify the genetic relationship of Chinese HIV-1 CRF01_AE strains with those circulating in other countries or regions globally. In particular, we focused on the linkage between CRF01_AE strains circulating among Chinese and United Kingdom populations, due to the availability of a large, diverse sequence database available in the United Kingdom (UK Collaborative HIV Cohort Steering Committee, 2004).

\section{MATERIALS AND METHODS}

\section{Sequence Databases}

HIV-1 CRF01_AE sequences covering the protease and partial reverse transcriptase coding regions $(\sim 1.0 \mathrm{~kb})$ were acquired from a public database, the Los Alamos HIV Sequence Database $^{1}$, and the UK HIV Drug Resistance Database $(\mathrm{UKHDRD})^{2}$ which is a centralized database of pol gene fragments generated from plasma samples collected throughout the United Kingdom as part of routine clinical care, largely during drug-naive chronic infection but also during acute infection and antiretroviral therapy failure. Sequence data are linked to demographic and clinical patient data held by the United Kingdom Collaborative HIV Cohort study (UK Collaborative HIV Cohort Steering Committee, 2004) and the national HIV/AIDS Reporting System database held at Public Health England (Public Health English, 2008). The London Multicenter Research Ethics Committee approved the use of the anonymous data in the UKHDRD. This collaborative project and its access to the database was approved by the UKHDRD Steering Committee.

\section{Query Sequences for Initial Blast Search}

The initial searching procedures used to screen and identify HIV-1 CRF01_AE strains associated with viral transmission in China from the above two databases are shown in Figure 1. Five predominant CRF01_AE clusters in China have been described previously (Feng et al., 2013). In order to make the query sequence more representative and avoid any potential bias due to genetically similar queries, three query sequences from three different regions, sampled at different collection dates and showing the largest genetic distance between each other within one cluster were selected for each of the five clusters. These 15 query sequences are as follows: FJ070010 (accession number JX112809), GD070010 (JX112819), and GX070003 (JX112829) for CN.01AE.HST/IDU-1 cluster; FJ070013 (JX112810), GX070005(JX112830), and JS070901 (JX112850) for CN.01AE.HST/IDU-2 cluster; GD070120 (JX112827), GZ070123 (JX112843), and FJ052 (EF036528) for CN.01AE.HST/IDU-3 cluster; CYM059 (JX112796), TJ070003(JX112859), and JS071101 (JX112853) for CN.01AE.MSM-4 cluster; CYM105 (JX112798), JL100005 (JX112846), and LN070008 (JX112854) for CN.01AE.MSM-5 cluster. An approximately $1.0 \mathrm{~kb}$ fragment from nucleotide 2253 to 3251 (corresponding to HIV-1 HXB2 sequence) in the pro-RT coding region of each query sequence was used for the Blast search.

\section{Initial Blast Search and Related Sequence Identification}

The online basic local alignment search tool (BLAST) ${ }^{3}$ was used to search the above two databases for all sequences which match the pro-RT fragment of any query sequence. For each Chinese

\footnotetext{
${ }^{1}$ www.hiv.lanl.gov

${ }^{2}$ www.hivrdb.org.uk

${ }^{3}$ http://blast.ncbi.nlm.nih.gov/
} 


\section{Flowchart}

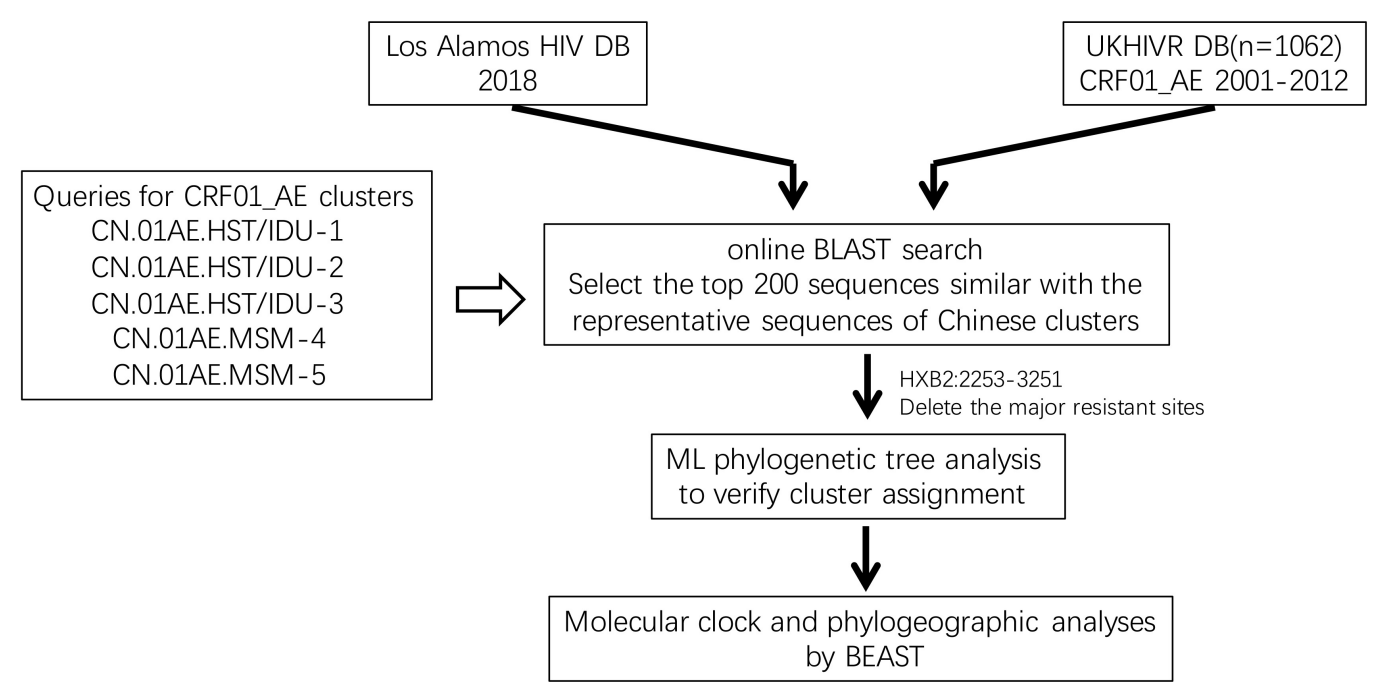

FIGURE 1 | The study flowchart used to screen and identify HIV-1 CRF01_AE strains associated with viral transmission in China. The Blast tool was used to search the sequence of interest in (i) the Los Alamos HIV sequence database and (ii) the United Kingdom HIV Drug Resistance Database. Five published Chinese major CRF01_AE clusters were used as queries (covering protease and partial reverse transcriptase nucleotide sequences). The top 200 sequences were selected and included in this analysis according to Blast scores. Phylogenetic and phylogeographic analyses were reconstructed to identify the relationship between Chinese CRF01_AE and CRF01_AE from other countries/regions globally.

CRF01_AE cluster, the first 200 sequences with the highest Blast score were selected from each of three query sequences. A total of 6000 sequences from two databases were selected for the five major CRF01_AE clusters. If there are duplicate sequences from an infected individual, only one of these sequences from this individual was retained for analysis. As recombination can affect the phylogenetic inference, so the recombinant signals of all sequences were analyzed in Recombination Detection Program 4 (RPD4) (Martin and Rybicki, 2000) before the reconstruction of phylogenetic tree. After removal of duplicates and recombinants, the retained sequences were used in phylogenetic analysis.

\section{Reconstruction of Phylogenetic Trees}

We reconstructed the phylogenetic relationship to confirm possible links between global sequences and Chinese CRF01_AE clusters. We also downloaded some reference sequences most of that were sampled during 1990s from the Los Alamos HIV sequence database, representing the primary geographical spread of CRF01_AE as follows: Africa (4 Central African Republic, 2 Cameroon, 1 Congo, 1 Gabon, and 1 Senegal), Europe (5 Switzerland, 2 France, 2 Sweden, 1 Germany and 1 Denmark), America (3 United States), South-east Asia (15 Thailand and 1 Vietnam), to fix the topology of phylogenetic tree. The phylogenetic trees were rooted using an outlier group containing three subtype $\mathrm{C}$ strains. The retained sequences after initial Blast search and 42 reference sequences were codon-aligned using the Gene Cutter tool (see footnote 3) and then manually edited using BioEdit 7.2. To mitigate the effect of antiretroviral therapy-related selection pressure on the phylogenetic analysis, all major HIV-1 drug resistance mutation sites were deleted according to the 2019 update of the drug resistance mutations in HIV published by the international AIDS Society, United States. ModelFinder package of IQ-TREE was used to judge the best fitting nucleotide substitution models and the maximumlikelihood (ML) tree was finally reconstructed under the generalized time reversible model of nucleotide substitution with gamma distribution for rate heterogeneity $(\mathrm{GTR}+\mathrm{I}+\mathrm{G})$ using IQ-TREE (version 1.6.12) (Nguyen et al., 2015). The branch support was estimated with the approximate likelihood-ratio (aLRT) SH-like test.

The access numbers of reference sequences downloaded from the Los Alamos HIV sequence database were as followed: AM040989, AF197340, AF197341, U51188, AY626951, GU207116, AM279456, AJ313417, AJ287011 in Africa, JF769840, KX691969, GQ848140, JF769833, GQ131600, AF347337, AM933273, AJ287043, AJ287053, AY165194, AY165218 in Europe, AY444803, AY444805, AY444806 in America, AF259954, U54771, AY713425, AF447828, AB220947, AF164485, AB220944, AB032741, AY713424, AY713419, AY125894, AY713422, AY945726, AY358040, AY945719, FJ185238 in South-east Asia, and AB023804, AB067155, AB254141 of subtype C.

\section{Molecular Clock and Phylogeographic Analyses}

The viral spatiotemporal trajectories were estimated through the time to Most Recent Common Ancestor and the localization of ancestors, using a Bayesian Markov Chain Monte Carlo (MCMC)-approach (BEAST v1.10.4 package), under GTR + I + G nucleotide substitution model. The sequences with 
available sampling date and locations that were identified to be associated with viral transmission in Chinese major CRF01_AE clusters were used to reconstruct the maximum clade credibility (MCC) tree. The posterior distribution was tested under a relaxed lognormal molecular clock, which has previously been shown to be more reliable in estimating viral phylogenies and divergence dates than "strict clock" and "non-clock" methods (Drummond et al., 2006). Several demographic models (parameter models: constant, exponential and logistic non-parameter models: skyline, skyride and skygrid) were compared using marginal likelihood estimators based on path sampling and stepping-stone sampling (PS/SS) analysis in BEAST v1.10.4, which would make sure the best fit model for the demographic signal was used. The MCMC chains were run for at least 200 million times and sampled every 20000 steps, for several times until getting the good convergence status. The output was analyzed in Tracer (version 1.5) and the related parameter estimates with an Effective Sample Size (ESS) over 200 were accepted. After the initial trees were summarized by TreeAnnotator (with $10 \%$ burn-in), the MCC tree was visualized and color-annotated with the FigTree (version 1.3). The median tMRCA was reported along with 95\% HPD intervals.

\section{Identification of Clusters}

The transmission clusters were initially estimated using a maximum-likelihood approach. Potential clusters were predefined as three or more sequences together in the reconstructed topology of the phylogenetic tree with the aLRT statistical support value of $>0.9$. Subsequently, clusters were re-supported at a posterior probability of 1.0 by Bayesian phylogenetic inference.

\section{RESULTS}

\section{The Global Dispersal of HIV-1 CRF01-AE Strains Associated With the Transmission in Mainland China}

After the initial search using 15 representative query sequences (three per cluster) in two sequence databases, 3850 duplicate sequences were removed from initial 6000 blast results and a large number of unique sequences $(n=2150)$ of HIV-1 CRF01_AE were retained for phylogenetic analysis, of which 1202 were Chinese sequences and 948 were from elsewhere (Supplementary Data Sheets 1, 2). Then, 1167 sequences from Mainland China and 269 sequences from elsewhere were identified to be possibly associated with the HIV-1 CRF01-AE transmission clusters in Mainland China, based on the topology of ML tree and >0.9 branch support values (Supplementary Figure S1 and Figure 2). Of these 269 foreign sequences, 212 were Asian sequences (181 from Vietnam, 18 from Japan, 9 from Hong Kong, 2 from Thailand, 2 from Malaysia) and 55 were European (48 from the United Kingdom, 4 from the Czechia, 1 from Germany, 1 from Sweden, 1 from Russia), and 2 were Australian (Table 1).

As shown in Table 1 and Figure 2, out of 269 sequences sampled outside Mainland China, 217 (80.7\%) matched CN.01AE.HST/IDU-2 cluster with the branch value of 0.92, but did not form any obvious epidemic sub-clusters. These included 180 strains from Vietnam, 36 from Europe (30 in the United Kingdom, 4 in Czechia, 1 in Sweden and 1 in Russia) and 1 strain from Australia, and most of them were sampled before 2010. All 30 UK CRF01-AE samples in CN.01AE.HST/IDU-2 were from non-white ethnic subjects. Most of them were heterosexuals or IDUs $(77 \%, 23 / 30)$, and were born in South or Southeast Asian countries (60\%, 18/30).

A total of $48(17.8 \%)$ of the 269 sequences sampled outside Mainland China, matched CN.01AE.MSM-4 and CN.01AE.MSM-5 clusters with the branch value of 0.98 and 0.97 (Figure 2). Within both of the two clusters circulating among MSM population in China, two distinct monophyletic sub-clusters were identified with good statistical support ( $\mathrm{SH}$ like value $=0.92$ and 0.93, respectively) as shown in Figure 2 . The sub-cluster located in CN.01AE.MSM-4 is consisted of 16 Japanese sequences sampled during 2009-2013, designated as JP-CN.01AE.MSM-4 sub-cluster (Figure 2). We noted that the remaining two sequences from Japan also belong to CN.01AE.MSM-4. Further, four United Kingdom sequences located within CN.01AE.MSM-4, and all of them were from MSM and two were born in East Asia. Another sub-cluster is within CN.01AE.MSM-5, consisted of three United Kingdom, five Hong Kong and one Thailand sequences, and because of the historical close link between United Kingdom and $\mathrm{HK}$, we designated it as UK-CN.01AE.MSM-5 sub-cluster (Figure 2). The three United Kingdom sequences were isolated in 2011-2012 from white males (MSM), while the five HK sequences were isolated in 2007-2008. Notably, other six United Kingdom heterosexual sequences were placed at the root of CN.01AE.MSM-5, most of which were white males (heterosexuals).

Additionally, our analysis also revealed that a few sequences outside mainland China (three from $\mathrm{HK}$ and one from Vietnam) belonged to CN.01AE.HST/IDU-1 and 3, which were prevalent among heterosexuals and IDUs in China (Table 1 and Figure 2).

\section{The Spatial-Temporal Scale of HIV-1 CRF01-AE Strains Associated With the Transmission in Mainland China}

To identify any export events of CRF01_AE strains related to Chinese major transmission clusters, we quantified the genetic divergence time in terms of the estimated time to the most recent common ancestor (tMRCA) using a Bayesian MCMC-based approach and also estimated the location of ancestor at the node of the tree. Many sequences (1172 from China and 226 from Vietnam) were identified to be associated with viral transmission in China through above phylogenetic analysis, and to reduce the computational burden in Bayesian running for spatialtemporal analysis, a sub-sampling procedure was performed that only one representative sequence was selected among high similarity clustered sequences (genetic distance $<2 \%$ ), using the online web server of CD-HIT-EST program (Huang et al., 2010; Eybpoosh et al., 2017). As a result, totally 328 sequences ( 186 from Mainland China, 56 from Vietnam, 88 from other countries/regions) and 42 references were used for the 


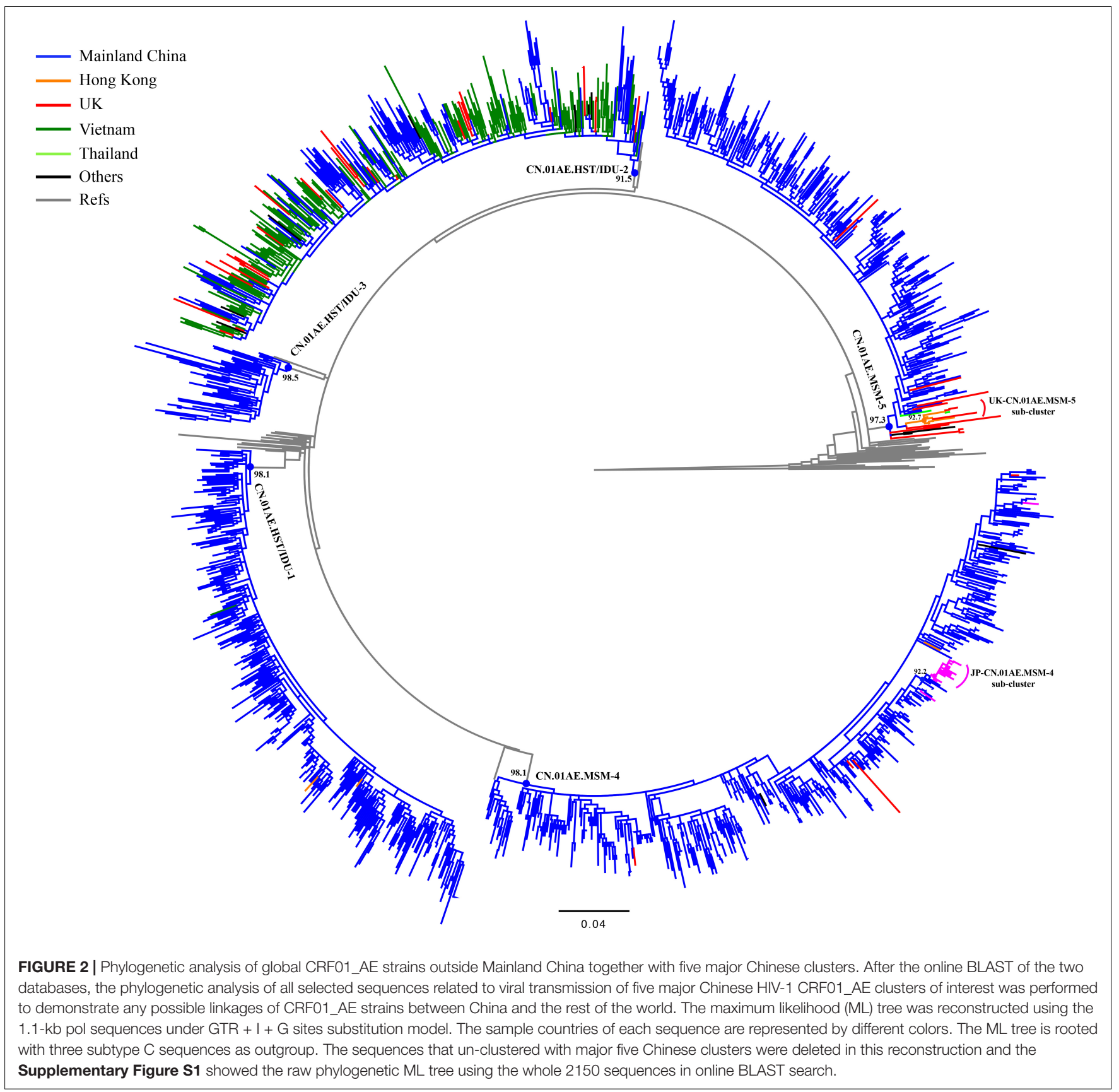

phylogeographic reconstruction. For this dataset, the Bayesian SkyGrid model was identified as the best fit coalescent tree prior after the comparison of different demographic models by a Bayes Factor (BF), using marginal likelihood estimators based on PS/SS analysis in BEAST v1.10.4 (Supplementary Table S1). As shown in Figure 3, the estimated tMRCAs of CN.01AE.HST/IDU-1, CN.01AE.HST/IDU-2 and CN.01AE.HST/IDU-3 were 1998.74 (95\% HPD interval 1996.00-2000.26), 1995.05 (1992.371996.16) and 1998.85 (1996.51-2000.56), respectively. The estimated tMRCAs of CN.01AE.MSM-4 and CN.01AE.MSM-5 were 1997.41 (1994.97-1999.48) and 1997.26 (1994.89-1999.53), respectively. The estimated tMRCAs of JP-CN.01AE.MSM-4 and
UK-CN.01AE.MSM-5 sub-clusters were 2006.76 (2002.942008.59) and 2001.91 (2000.26-2003.53), respectively. Remarkably, six United Kingdom heterosexual strains and one Malaysian strain were seen at the root within CN.01AE.MSM-5, and the inner cluster of Chinese MSM strains was placed inside these six United Kingdom strains, supported with a posterior probability value of 0.89 , and the estimated tMRCA of 1998.85 .

For either ML tree or MCC tree, African strains were at the root and Thailand strains were highly centralized outside Chinese clusters, and European-American strains dispersed across the tree. The divergence times of African and Asian CRF01-AE strains were $1978.11(1971.39-1982.93)$ and 1984.03 


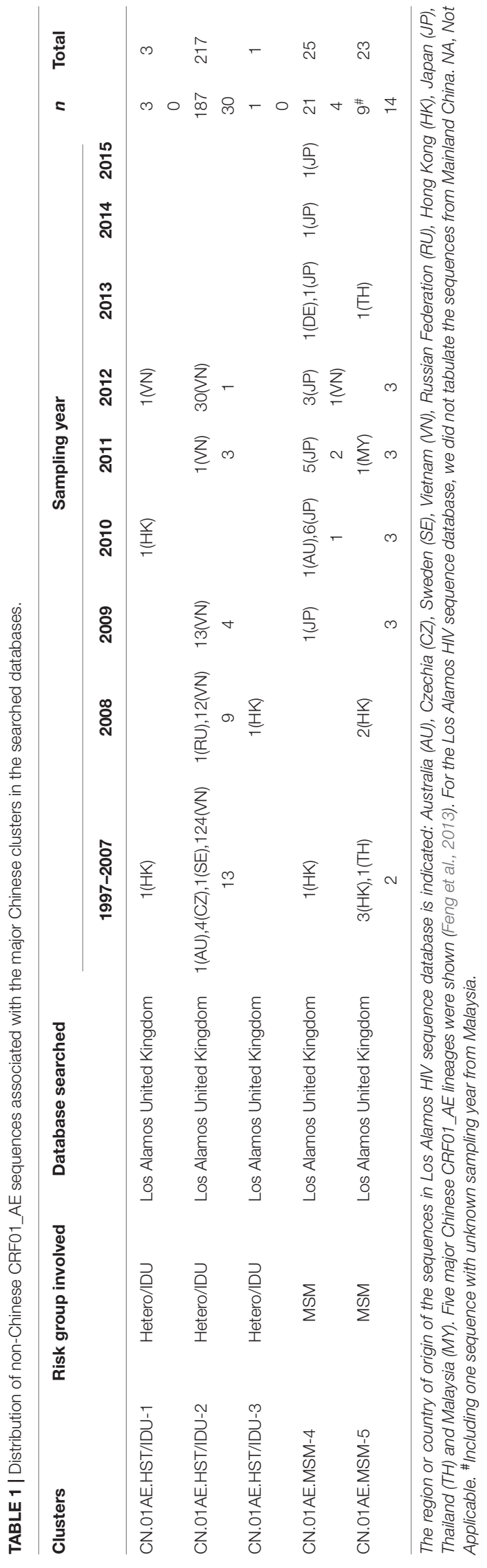

(1981.92-1986.31). This is in agreement with the hypothesis that HIV-1 CRF01_AE originated from Africa and then migrated to Thailand, from which, as the secondary source, the virus subsequently, spread to most areas worldwide (Angelis et al., 2015).

\section{DISCUSSION}

In recent years, HIV-1 non-B subtypes increased rapidly, not only in Asia, but also in Europe-America where HIV-1 subtype B is generally dominating. In Mainland China, HIV-1 CRF01_AE has caused a large epidemic, and several distinct clusters related to transmission among various high-risk populations have been identified. This study is the first detailed analysis of the possible dissemination of major HIV-1 CRF01_AE clusters from Mainland China to other countries or regions.

Angelis et al. (2015) reported that China was a sink and CRF01_AE strains were mainly imported from neighboring countries and then evolved into "distinct" Chinese strains, based on a global dataset of $2736 \mathrm{CRF} 01-\mathrm{AE}$ sequences acquired from many public databases and cohort studies. Our results showed that although major HIV-1 CRF01_AE clusters in China were imported from Thailand, one of which might have entered China via Vietnam (CN.01AE.HST/IDU-2 cluster) (Figures 2, 3) (Liao et al., 2009), and these Chinese HIV-1 CRF01_AE strains had spread to other countries/regions.

After using 15 representative query sequences for initial blast screening and further phylogenetic analysis, 269 samples from other countries or regions matched the five major CRF01_AE clusters associated with HIV transmission in China. Among these, $80.7 \%$ (217/269) belong to CN.01AE.HST/IDU-2 cluster, most of which were sampled in Vietnam, and the rest from the United Kingdom or other countries. None of these 217 samples formed any distinct sub-clusters within CN.01AE.HST/IDU2 cluster (Figures 2, 3), and most were sampled before 2010 (Table 1). Therein, of 30 individuals diagnosed in the United Kingdom as HIV-1, 18 are with a South/Southeast Asia origin and 23 were infected via heterosexual contact or drug injection (Table 2). The molecular clock analysis indicated that the tMRCA of CN.01AE.HST/IDU-2 was 1995, earlier than other four Chinese clusters (Figure 3). It is known, many Vietnamese started to immigrate to the United Kingdom since the end of Vietnam War in 1975, and because the Vietnamese communities in the United Kingdom were very small and closed, it was hard to integrate themselves further to the host communities (Fakoya et al., 2015; Vietnamese people in the United Kingdom). We infer that these HIV-1 CRF01_AE strains that own a common ancestor with Chinese CRF01_AE strains, circulating among Asian communities in Europe might be brought in by Vietnamese immigrants around mid-1990, and due to the closed feature of Vietnamese communities in the United Kingdom, the strains did not enter the local British communities. Similarly, six samples from the UK Drug Resistance Database were seen at the root of CN.01AE.MSM5 with high support value (Figures 2, 3). Four of six were born in the United Kingdom and two were born in Southeast 


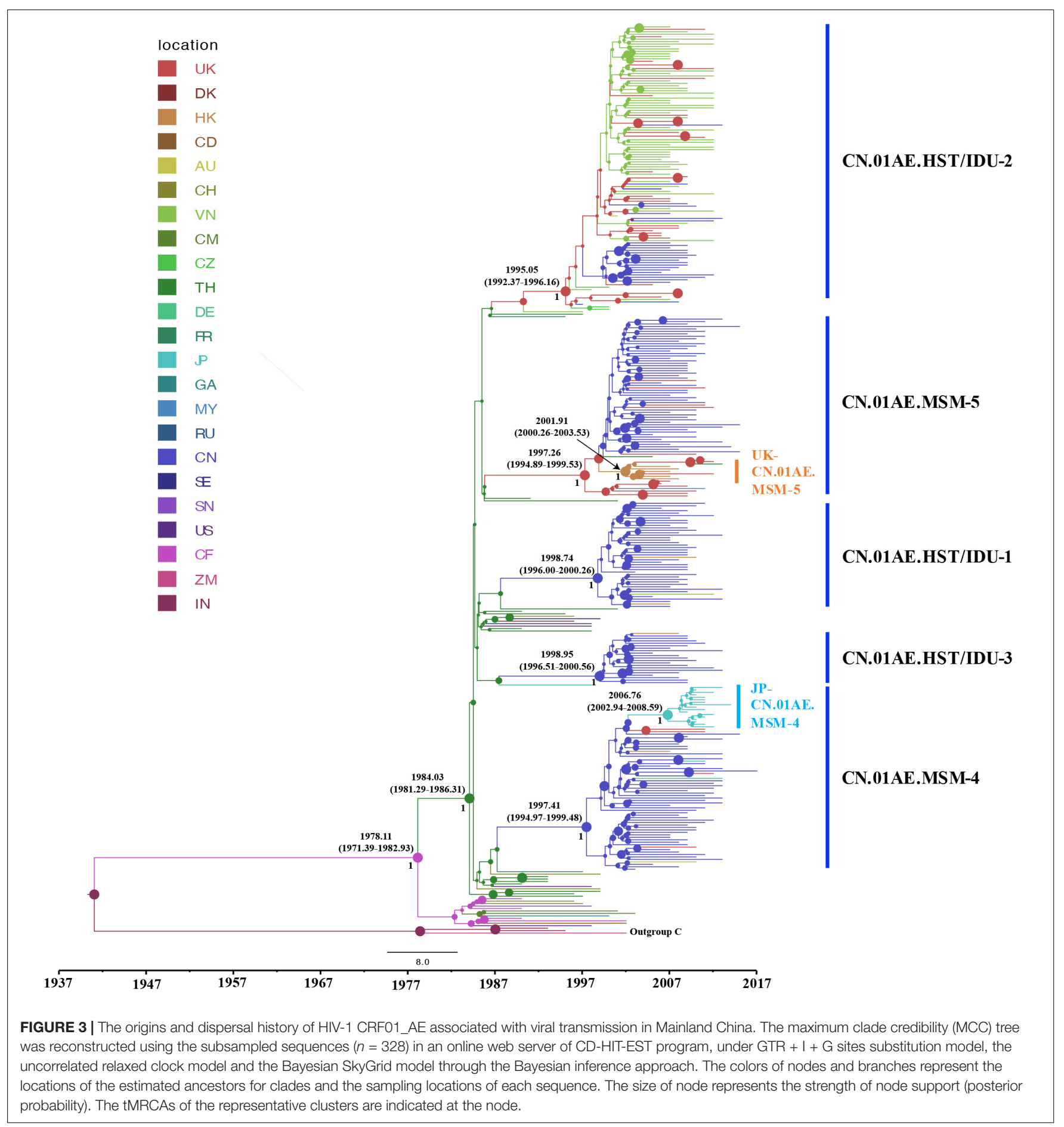

Asia, and all the six United Kingdom individuals were infected via heterosexual contact (Table 2). The divergence time of CN.01AE.MSM-5 was $\sim 1997$ (Figure 3). Due to Thailand's sex industry and the popular international tourism to Thailand during the last three decades (Angelis et al., 2015), the CRF01_AE strains associated with CN.01AE.MSM-5 might be brought back to the United Kingdom initially by returning tourists who were infected via heterosexual contact in Southeast Asia. Although the estimated locations of ancestors of CN.01AE.HST/IDU-2 and CN.01AE.MSM-5 were both in the United Kingdom as shown in phylogeographic tree (Figure 3), we inferred these United Kingdom individuals were most likely infected with Southeast Asian CRF01_AE, such as Thailand or Vietnam, and so the ancestors of these two clusters should still be located in Southeast Asia. It is therefore suggested that the CRF01_AE strains associated with viral transmission in 
TABLE 2 | Summary of epidemiologic and demographic information of subjects in the UK HIV Drug Resistance Database who were infected with HIV-1 CRF01_AE strains associated with Chinese clusters.

\begin{tabular}{|c|c|c|c|c|c|c|c|}
\hline CN cluster & Sample ID & Age Range* & Sex & Ethnicity/Nationality & Risk factor & Country of origin/birth & Sampling year \\
\hline \multirow[t]{30}{*}{ CN.01AE.HST/IDU-2 } & 11UK.C2.01 & $B$ & Male & Unknown & Heterosexual & South and South East Asia & 2011.03 \\
\hline & 09UK.C2.02 & $E$ & Male & Other Asian/Oriental & IDU & Unknown & 2009.04 \\
\hline & 11UK.C2.03 & B & Unknown & Unknown & Unknown & Unknown & 2011.01 \\
\hline & 09UK.C2.04 & Unknown & Unknown & Unknown & Unknown & Unknown & 2009.04 \\
\hline & 09UK.C2.05 & $\mathrm{B}$ & Male & Other Asian/Oriental & Homo/bisexual & South and South East Asia & 2009.05 \\
\hline & 06UK.C2.06 & $A$ & Male & Other Asian/Oriental & Heterosexual & South and South East Asia & 2006.04 \\
\hline & 09UK.C2.07 & C & Male & Other & IDU & Unknown & 2009.06 \\
\hline & 08UK.C2.08 & $\mathrm{D}$ & Male & Other Asian/Oriental & IDU & South and South East Asia & 2008.09 \\
\hline & 06UK.C2.09 & C & Female & Other Asian/Oriental & Heterosexual & South and South East Asia & 2006.11 \\
\hline & 07UK.C2.10 & $\mathrm{C}$ & Male & Other & Heterosexual & South and South East Asia & 2007.01 \\
\hline & 08UK.C2.11 & C & Male & Other/Mixed & Heterosexual & South and South East Asia & 2008.07 \\
\hline & 08UK.C2.12 & $\mathrm{C}$ & Female & Other Asian/Oriental & Heterosexual & South and South East Asia & 2008.04 \\
\hline & 08UK.C2.13 & $\mathrm{C}$ & Male & Other Asian/Oriental & other & South and South East Asia & 2008.11 \\
\hline & 07UK.C2.14 & B & Male & Other Asian/Oriental & IDU & South and South East Asia & 2007.05 \\
\hline & 06UK.C2.15 & C & Male & Other Asian/Oriental & IDU & United Kingdom & 2006.12 \\
\hline & 12UK.C2.16 & B & Male & Other Asian/Oriental & Heterosexual & Unknown & 2012.01 \\
\hline & 07UK.C2.17 & $\mathrm{C}$ & Female & Other Asian/Oriental & Heterosexual & South and South East Asia & 2007.08 \\
\hline & 05UK.C2.18 & C & Male & Other Asian/Oriental & IDU & South and South East Asia & 2005.07 \\
\hline & 11UK.C2.19 & Unknown & Male & Other Asian/Oriental & Heterosexual & South and South East Asia & 2011.06 \\
\hline & 05UK.C2.20 & $\mathrm{D}$ & Unknown & Unknown & Unknown & Unknown & 2005.09 \\
\hline & 05UK.C2.21 & $\mathrm{C}$ & Male & Black-other/unspecified & Heterosexual & South and South East Asia & 2005.07 \\
\hline & 08UK.C2.22 & $B$ & Male & Other Asian/Oriental & Homo/bisexual & South and South East Asia & 2008.03 \\
\hline & 08UK.C2.23 & $\mathrm{B}$ & Male & Other Asian/Oriental & Heterosexual & South and South East Asia & 2010.10 \\
\hline & 05UK.C2.24 & $\mathrm{C}$ & Male & Other/Mixed & Heterosexual & United Kingdom & 2005.08 \\
\hline & 08UK.C2.25 & C & Male & Other/Mixed & Heterosexual & South and South East Asia & 2008.05 \\
\hline & 06UK.C2.26 & Unknown & Male & Other Asian/Oriental & Heterosexual & South and South East Asia & 2006.07 \\
\hline & 08UK.C2.27 & B & Unknown & Unknown & Unknown & Unknown & 2008.08 \\
\hline & 08UK.C2.28 & $\mathrm{F}$ & Male & Other & Heterosexual & Caribbean & 2008.09 \\
\hline & 05UK.C2.29 & C & Male & Other Asian/Oriental & IDU & Unknown & 2005.02 \\
\hline & 05UK.C2.30 & $\mathrm{D}$ & Male & Black-African & Heterosexual & Southern Africa & 2005.06 \\
\hline \multirow[t]{4}{*}{ CN.01AE.MSM-4 } & 10UK.C4.01 & $\mathrm{D}$ & Male & White & Homo/bisexual & United Kingdom & 2010.04 \\
\hline & HUK.C4.02 & $B$ & Male & Other Asian/Oriental & Homo/bisexual & East Asia & 2011.05 \\
\hline & 11UK.C4.03 & $\mathrm{C}$ & Male & White & Homo/bisexual & Southern Africa & 2011.02 \\
\hline & 12UK.C4.04 & $\mathrm{B}$ & Male & Other Asian/Oriental & Homo/bisexual & East Asia & 2012.02 \\
\hline \multirow[t]{14}{*}{ CN.01AE.MSM-5 } & 12UK.C5.01 & $\mathrm{B}$ & Male & White & Homo/bisexual & United Kingdom & 2012.06 \\
\hline & 11UK.C5.02 & $\mathrm{D}$ & Male & White & Homo/bisexual & Unknown & 2011.08 \\
\hline & 12UK.C5.03 & B & Male & White & Homo/bisexual & North Africa and Middle East & 2012.11 \\
\hline & 12UK.C5.04 & $\mathrm{C}$ & Male & White & Homo/bisexual & United Kingdom & 2012.03 \\
\hline & 11UK.C5.05 & B & Male & Other Asian/Oriental & Homo/bisexual & East Asia & 2011.02 \\
\hline & 10UK.C5.06 & B & Male & White & Homo/bisexual & Unknown & 2010.05 \\
\hline & 11UK.C5.07 & $\mathrm{D}$ & Male & White & Heterosexual & United Kingdom & 2011.08 \\
\hline & 10UK.C5.08 & $\mathrm{C}$ & Male & Other/Mixed & Heterosexual & East Asia & 2010.09 \\
\hline & 06UK.C5.09 & $B$ & Male & White & Heterosexual & United Kingdom & 2006.05 \\
\hline & 09UK,C5.10 & $\mathrm{C}$ & Male & White & Heterosexual & United Kingdom & 2009.09 \\
\hline & 07UK.C5.11 & B & Female & Other/Mixed & Heterosexual & South and South East Asia & 2007.09 \\
\hline & 09UK.C5.12 & $E$ & Male & White & Heterosexual & United Kingdom & 2009.08 \\
\hline & 09UK.C5.13 & $\mathrm{F}$ & Male & White & Heterosexual & South and South East Asia & 2009.09 \\
\hline & 10UK.C5.14 & $\mathrm{C}$ & Male & White & Heterosexual & United Kingdom & 2010.06 \\
\hline
\end{tabular}

*Age Range: A (<20); B (20-29); C (30-39); D (40-49); E (50-59); F (60-69).

China (CN.01AE.HST/IDU-2 and CN.01AE.MSM-5) were likely introduced to the United Kingdom initially by the Vietnamese immigrants and also returning tourists from Southeast Asia around the middle 1990, through the routes of heterosexual contact and/or drug injection. These CRF01_AE strains also spread to China around the same time from Southeast Asia 


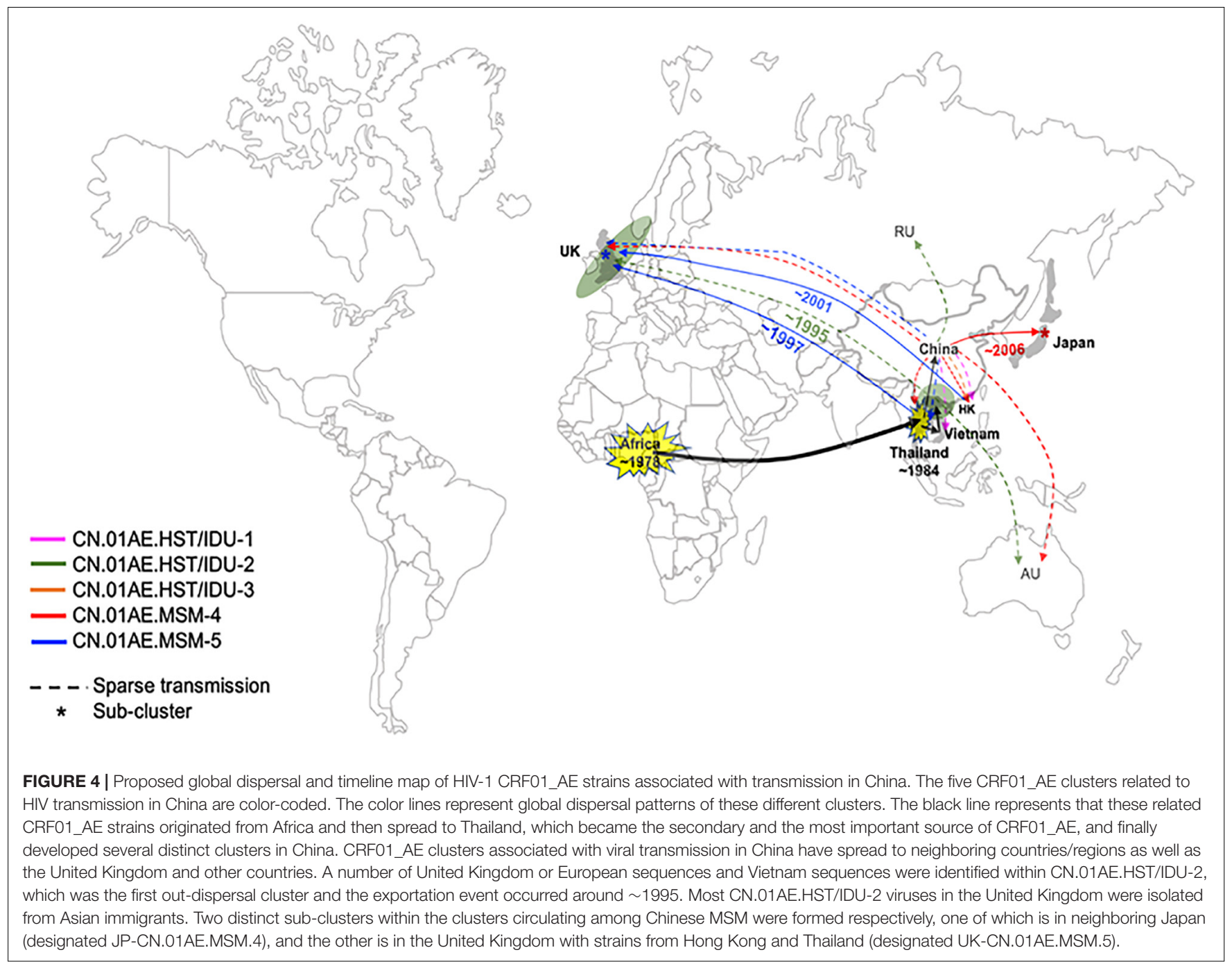

and then formed two distinct clusters. Although Chinese sequences and foreign sequences within CN.01AE.HST/IDU-2 and CN.01AE.MSM-5 shared the same common ancestor, they did not have direct transmission relationship.

Interestingly, we found that three United Kingdom MSM sequences, five $\mathrm{HK}$ sequences and one Thailand sequence were placed together as another distinct sub-cluster within CN.01AE.MSM-5. The tMRCAs of CN.01AE.MSM-5, the inner node (containing Chinese samples) and the sub-cluster UKCN.01AE.MSM-5 was 1997, 1998, and 2001, respectively (Figure 3). Similarly, a distinct sub-cluster consisting of Japanese sequences was formed within CN.01AE.MSM-4, with low genetic distances (Figure 2). The molecular clock results showed that the tMRCAs for CN.01AE.MSM-4 and sub-cluster JPCN.01AE.MSM-4 were 1997 and 2006, respectively (Figure 3). Although previous researchers generally agreed that Japan also played a secondary role in the global epidemic of HIV-1 CRF01_AE strains (Shiino et al., 2014; Angelis et al., 2015), our results indicate that Chinese CRF01_AE strains (CN.01AE.MSM4) have expanded to Japan around 2006, a little earlier than that reported by Kondo et al. (2013), and possibly caused a potential epidemic in Japan. Of course, some sequences from other countries are also scattered in CN.01AE.MSM-4 and 5. Taken these results together, it suggests that CRF01_AE strains of CN.01AE.MSM-4 and CN.01AE.MSM-5 have been circulating among Chinese MSM for several years, and then dispersed outside, not only to the neighboring countries, but also to the United Kingdom or other countries. Based on our results and previous report (Angelis et al., 2015), we proposed a global migration route map of CRF01_AE clusters circulating in Asia and China (Figure 4).

The major limitations of this study are that (1) only partial sequences $(p o l)$ were available for this retrospective analysis, (2) the sequences were from only two HIV databases available to this analysis and the sampling of global CRF01_AE strains was less representative, and (3) the sampling periods of the sequences from Los Alamos database and UKHDRD were different, which might influence the investigation of the dispersal patterns.

In conclusion, our analysis suggests that HIV CRF01_AE strains related to viral transmission in China were initially 
brought to the United Kingdom or other countries by Asian immigrants or international tourists back from Southeast Asia during the 1990s via heterosexuals and IDUs, and then after having circulated among Chinese MSM for several years, they dispersed outside again, possibly through MSM network. This study provided evidence of regional and global dispersal of Chinese CRF01-AE strains. It would also help understand the global landscape of HIV epidemic associated with CRF01-AE transmission and highlight the need for further international collaborative study in this field.

\section{DATA AVAILABILITY STATEMENT}

All datasets generated for this study are included in the article/Supplementary Material.

\section{AUTHOR CONTRIBUTIONS}

HS and $\mathrm{HZ}$ conceived and designed the study. $\mathrm{XH}$ and $\mathrm{BZ}$ performed data collection. MA performed data analysis and wrote the first draft. HS, HZ, XH, SF, and SE contributed in the process of study design, analyzing data, and writing the manuscript.

\section{FUNDING}

This work was supported by the Mega-projects of National Science Research for the 13th Five-Year Plan (2017ZX10201101), the National Natural Science Foundation of China (81401655), Central Public-Interest Scientific Institution Basal Research Fund

\section{REFERENCES}

Abubakar, Y. F., Meng, Z. F., Zhang, X. Y., and Xu, J. Q. (2013). Multiple independent introductions of HIV-1 CRF01_AE Identified in China: what are the implications for prevention? Plos One 8:e080487. doi: 10.1371/journal.pone. 0080487

Angelis, K., Albert, J., Mamais, I., Magiorkinis, G., Hatzakis, A., and Hamouda, O. (2015). Global dispersal pattern of HIV Type 1 Subtype CRF01_AE: a genetic trace of human mobility related to heterosexual sexual activities centralized in Southeast Asia. J. Infect. Dis. 211, 1735-1744. doi: 10.1093/infdis/jiu666

Beloukas, A., Psarris, A., Giannelou, P., Kostaki, E., Hatzakis, A., and Paraskevis, D. (2016). Molecular epidemiology of HIV-1 infection in Europe: an overview. Infect. Genet. Evol. 46, 180-189. doi: 10.1016/j.meegid.2016. 06.033

Cuevas, M., Fernandez-Garcia, A., Sanchez-Garcia, A., Gonzalez-Galeano, M., Pinilla, M., and Sanchez-Martinez, M. (2009). Incidence of non-B subtypes of HIV-1 in Galicia, Spain: high frequency and diversity of HIV-1 among men who have sex with men. Euro. Surveill. 14:19413.

Dennis, A. M., Hue, S., Learner, E., Sebastian, J., Miller, W. C., and Eron, J. J. (2017). Rising prevalence of non-B HIV-1 subtypes in North Carolina and evidence for local onward transmission. Virus Evol. 3:vex013. doi: 10.1093/ve/vex013

Drummond, A. J., Ho, S. Y., Phillips, M. J., and Rambaut, A. (2006). Relaxed phylogenetics and dating with confidence. PLoS Biol. 4:e88. doi: 10.1371/ journal.pbio.0040088

Eybpoosh, S., Bahrampour, A., Azadmanesh, K., Mostafavi, E., Haghdoost, A. A., and Zolala, F. (2017). Spatio-temporal dynamics of HIV-I Subtype B and
(2018PT31042), and Development Programme of the Innovative Group by Ministry of Education (2016). The UK HIV Drug Resistance Database was supported by the UK Medical Research Council (Award Number 164587).

\section{ACKNOWLEDGMENTS}

We thank David Dunn and Andrew Leigh-Brown for comments on the manuscript, and the UK HIV Drug Resistance Database for the contribution of sequence data. A full listing of contributors to UKHDRD is described at www.hivrdb.org.uk.

\section{SUPPLEMENTARY MATERIAL}

The Supplementary Material for this article can be found online at: https://www.frontiersin.org/articles/10.3389/fmicb. 2020.00061/full\#supplementary-material

FIGURE S1 | The reconstructed raw phylogenetic ML tree using all associated sequences after initial BLAST search. Out of 2150 unique sequences from the initial BLAST search, 983 sequences were not clustered with HIV-1 CRF01_AE strains associated with viral transmission in Mainland China. Black branches represent un-clustered sequences from BLAST search, and gray branches represent 42 reference sequences downloaded from the Los Alamos HIV sequence database. The $M L$ tree is rooted with 3 subtype $C$ sequences as outgroup.

TABLE S1 | The best fit tree prior for the dataset in Bayesian analysis.

DATA SHEET S1 | The alignment of 2150 unique sequences without major drug resistance sites screened after the initial online blast, which was used to reconstruct the phylogenetic tree in this study.

DATA SHEET S2 | The raw alignment of 2150 unique sequences after the initial online blast.

circulating recombinant form 01_AE Clades in Iran: a phylogeographic approach. Iran. Red Crescent Med. J. 19:e0156499.

Fakoya, I., Alvarez-del Arco, D., Woode-Owusu, M., Monge, S., RiveroMontesdeoca, Y., and Delpech, V. (2015). A systematic review of postmigration acquisition of HIV among migrants from countries with generalised HIV epidemics living in Europe: implications for effectively managing HIV prevention programmes and policy. Bmc Public Health 15:561.

Feng, Y., He, X., Hsi, J. H., Li, F., Li, X., and Wang, Q. (2013). The rapidly expanding CRF01_AE epidemic in China is driven by multiple lineages of HIV1 viruses introduced in the 1990s. AIDS 27, 1793-1802. doi: 10.1097/QAD. 0b013e328360db2d

Fox, J., Castro, H., Kaye, S., McClure, M., Weber, J. N., and Fidler, S. (2010). Epidemiology of non-B clade forms of HIV-1 in men who have sex with men in the UK. Aids 24, 2397-2401. doi: 10.1097/QAD.0b013e32833cbb5b

Hawke, K. G., Waddell, R. G., Gordon, D. L., Ratcliff, R. M., Ward, P. R., and Kaldor, J. M. (2013). HIV non-B subtype distribution: emerging trends and risk factors for imported and local infections newly diagnosed in South Australia. AIDS Res. Hum. Retroviruses 29, 311-317. doi: 10.1089/aid.2012. 0082

Hemelaar, J. (2013). Implications of HIV diversity for the HIV-1 pandemic. J. Infect. 66, 391-400. doi: 10.1016/j.jinf.2012.10.026

Huang, Y., Niu, B., Gao, Y., Fu, L., and Li, W. (2010). CD-HIT Suite: a web server for clustering and comparing biological sequences. Bioinformatics 26, 680-682. doi: 10.1093/bioinformatics/btq003

Kondo, M., Lemey, P., Sano, T., Itoda, I., Yoshimura, Y., and Sagara, H. (2013). Emergence in Japan of an HIV-1 variant associated with transmission 
among men who have sex with men (MSM) in China: first indication of the International Dissemination of the Chinese MSM lineage. J. Virol. 87, 5351-5361. doi: 10.1128/JVI.02370-12

Liao, H., Tee, K. K., Hase, S., Uenishi, R., Li, X. J., Kusagawa, S., et al. (2009). Phylodynamic analysis of the dissemination of HIV-1 CRF01_AE in Vietnam. Virology 391, 51-56. doi: 10.1016/j.virol.2009. 05.023

Martin, D., and Rybicki, E. (2000). RDP: detection of recombination amongst aligned sequences. Bioinformatics 16, 562-563. doi: 10.1093/bioinformatics/16. 6.562

Neogi, U., Haggblom, A., Santacatterina, M., Bratt, G., Gisslen, M., Albert, J., et al. (2014). Temporal trends in the Swedish HIV-1 epidemic: increase in non-B subtypes and recombinant forms over three decades. PLoS One 9:e99390. doi: 10.1371/journal.pone.0099390

Nguyen, L. T., Schmidt, H. A., von Haeseler, A., and Minh, B. Q. (2015). IQ-TREE: a fast and effective stochastic algorithm for estimating maximumlikelihood phylogenies. Mol. Biol. Evol. 32, 268-274. doi: 10.1093/molbev/ msu300

Nikolopoulos, G. K., Kostaki, E. G., and Paraskevis, D. (2016). Overview of HIV molecular epidemiology among people who inject drugs in Europe and Asia. Infect. Genet. Evol. 46, 256-268. doi: 10.1016/j.meegid.2016. 06.017

Peeters, M., Jung, M., and Ayouba, A. (2013). The origin and molecular epidemiology of HIV. Expert. Rev. Anti. Infect. Ther. 11, 885-896. doi: 10.1586/ 14787210.2013.825443

Peng, X. R., Wu, H. B., Peng, X. M., Jin, C. Z., and Wu, N. P. (2015). Heterogeneous Evolution of HIV-1 CRF01_AE in Men Who Have Sex with Men (MSM) and Other Populations in China. Plos One 10:e0143699. doi: 10.1371/journal.pone. 0143699

Public Health English (2008). National HIV/AIDS Reporting System (HARS).

Shiino, T., Hattori, J., Yokomaku, Y., Iwatani, Y., Sugiura, W., and Japanese Drug Resistance Hiv-1 Surveillance Network. (2014). Phylodynamic Analysis Reveals CRF01_AE Dissemination between Japan and Neighboring Asian countries and the role of intravenous drug use in transmission. PLoS One 9:e102633. doi: 10.1371/journal.pone.0102633

Su, Y., Liu, H., Wu, J., Zhu, L., and Wang, N. (2014). Distribution of HIV-1 genotypes in China: a systematic review. Zhonghua Liu Xing Bing Xue Za Zhi. $35,1164-1168$.
Sullivan, P. S., Hamouda, O., Delpech, V., Geduld, J. E., Prejean, J., Semaille, C., et al. (2009). Reemergence of the HIV epidemic among men who have sex with men in North America. Western Europe, and Australia, 1996-2005. Ann. Epidemiol. 19, 423-431. doi: 10.1016/j.annepidem.2009. 03.004

Thomson, M. M., and Najera, R. (2007). Increasing HIV-1 genetic diversity in Europe. J. Infect. Dis. 196, 1120-1124. doi: 10.1086/521683

UK Collaborative HIV Cohort Steering Committee, (2004). The creation of a large UK-based multicentre cohort of HIV-infected individuals: The UK Collaborative HIV Cohort (UK CHIC) Study. HIV Med. 5, 115-124. doi: 10.1111/j.1468-1293.2004.00197.x

UK Collaborative Group on HIV Drug Resistance (2014). The increasing genetic diversity of HIV-1 in the UK, 2002-2010. Aids 28, 773-780. doi: 10.1097/Qad. 0000000000000119

Vietnamese people in the United Kingdom. Vietnamese people in the United Kingdom. Avaliable at: https://en.wikipedia.org/wiki/Vietnamese_ people_in_the_United_Kingdom. (accessed October 27, 2019).

Wang, X., He, X., Zhong, P., Liu, Y., Gui, T., and Jia, D. (2017). Phylodynamics of major CRF01_AE epidemic clusters circulating in mainland of China. Sci. Rep. 7:6330. doi: 10.1038/s41598-017-06573-6

Zeng, H., Li, T., Wang, Y., Sun, B., and Yang, R. (2016). The Epidemic Dynamics of Four Major Lineages of HIV-1 CRF01_AE Strains After Their Introduction into China. AIDS Res. Hum. Retroviruses 32, 420-426. doi: 10.1089/AID.2015.0212

Zhang, L., Wang, Y. J., Wang, B. X., Yan, J. W., Wan, Y. N., and Wang, J. (2015). Prevalence of HIV-1 subtypes among men who have sex with men in China: a systematic review. Int. J. Std. Aids 26, 291-305. doi: 10.1177/0956462414543841

Conflict of Interest: The authors declare that the research was conducted in the absence of any commercial or financial relationships that could be construed as a potential conflict of interest.

Copyright (C) 2020 An, Han, Zhao, English, Frost, Zhang and Shang. This is an open-access article distributed under the terms of the Creative Commons Attribution License (CC BY). The use, distribution or reproduction in other forums is permitted, provided the original author(s) and the copyright owner(s) are credited and that the original publication in this journal is cited, in accordance with accepted academic practice. No use, distribution or reproduction is permitted which does not comply with these terms. 